\title{
Foundations of Lexicographic Cooperative Game Theory
}

\author{
Guram N. Beltadze \\ Department of Control Systems, Georgian Technical University, Georgia, Tbilisi \\ Email: gbeltadze@yahoo.com
}

\begin{abstract}
In the article $m$ dimensional lexicographic noncooperative games $\Gamma^{L}=\left(\Gamma^{1}, \ldots, \Gamma^{m}\right)$ are defined for the players' $N=\{1,2, \ldots, n\}$ for which there exists a characteristic function $v=\left(v^{1}, v^{2}, \ldots, v^{m}\right)^{T}$. Some main features are proved of $v$ function in a lexicographic case. A lexicographic cooperative game is called a couple $\langle N, v\rangle$, where $v$ is a real vectorfunction on $N$ subsets and the following conditions are

fulfilled

$v(\varnothing)=\mathrm{O} ; v(T \cup S) \succcurlyeq{ }^{L} v(T)+v(S), T, S \subseteq N$,

$T \cap S=\varnothing$. Such cooperative game is denoted by $v=\left(v^{1}, v^{2}, \ldots, v^{m}\right)^{T} . \quad X$ imputation and its set $E(v)$ is defined in $v$ game. It is proved that $E(v)$ is nonempty and its full characterisation is given. Domination over $E(v)$ set is defined. Thus, the main foundations are given, according to this, it is possible to explore the main principles of optimality.
\end{abstract}

IndexTerms - Game, Lexicography, Noncooperative Game, Cooperative Game, Imputation, Domination

\section{INTRODUCTION}

In a classical game theory the main basic model is a noncooperative game that is defined by

$$
\Gamma=<N,\left\{X_{i}\right\}_{i \in N},\left\{H_{i}\right\}_{i \in N}>
$$

system,where $N=\{1,2, \ldots, n\}$ is a set of players', $X_{i}$ is $i \in N$ player's set of mixed strategies,

$$
H_{i}: \prod_{i \in N} X_{i}=X \rightarrow R^{1}
$$

is $i \in N$ player's real-valued payoff function, it tries to maximize the function. It means that, $\Gamma$ game is finite i.e. the players' sets of pure strategies are finite. In $\Gamma$ game players choose their strategies independently $x_{i} \in X_{i}, i \in N$ and we get a situation $x=\left(x_{1}, x_{2}, \ldots, x_{n}\right) \in X$. Players payoff functions $H_{i}(x), i \in N$ are defined for every $x \in X$.
In $\Gamma$ game players simultaneously and independently choose their strategies so that they don't inform each other about it. Therefore $\Gamma$ game is called a noncooperative or noncoalition. Players strategic behaviours are studied in such games, with the help of such strategies they get this or that kind of guaranteed payoffs (utilities). Hence, the task is to find such kind of situation $x^{*} \in X$, that will be multicriteria $\max _{x}\left(H_{1}(x), \ldots, H_{m}(x)\right) \quad$ problem solution.

About classical cooperative games, we can say that players' cooperative behaviors are studied in $\Gamma$ game's condition. Here players can enrol in coalition and each member of a coalition can negotiate with the rest for choosing their strategies consistently. At the same time players can sum up their payoffs and then they will imputate them. In the definition of a classical cooperative game we mean that players payoffs have transferring property or payoffs are measured by a unique scalar, and they can transfer from one player to another without limitation.

Formally, a classical cooperative game is called a couple $\langle N, v\rangle$, where $N=\{1,2, \ldots, n\}$ is a set of players, and $v: 2^{N} \rightarrow R^{1}$ is a real-valued function, that is defined on $N$ every subset, also it means that $v(\varnothing)=0$. A subset $S \subseteq N$ is called a coalition and $v$ function is $\Gamma$ game's characteristic function. Therefore in cooperative $\langle N, v\rangle$ game the imputation will be appeared by $n$ - vector's real components.

Let us denote that in

$\Gamma=<N,\left\{X_{i}\right\}_{i \in N},\left\{H_{i}\right\}_{i \in N}>$

game $H_{i}=\left(H_{i}^{1}, H_{i}^{2}, \ldots, H_{i}^{m}\right)$ is a vector-function of $i \in N$ player's payoff, for every $i \in N$ vector $H_{i}$ has the same dimensional $m$ and on the set of situations $\quad X=\prod_{i \in N} X_{i} \quad$ their comparison holds lexicographically. We call such game a lexicographic noncooperative (noncoalition) game with dimensional $m$ and we note it in the following way 


$$
\Gamma^{L}=<N,\left\{X_{i}\right\}_{i \in N},\left\{H_{i}\right\}_{i \in N}>\equiv\left(\Gamma^{1}, \ldots, \Gamma^{m}\right)
$$

For two $a=\left(a_{1}, \ldots, a_{m}\right)$ and $b=\left(b_{1}, \ldots, b_{m}\right)$ vectors lexicographic preference $a \succ^{L} b$ means that it fulfills one of the following $m$ conditions:

1) $a_{1}>b_{1}$;2) $a_{1}=b_{1}, a_{2}>b_{2} ; . . . ; m$ ) $a_{1}=b_{1}, \ldots, a_{m-1}=b_{m-1}, a_{m}>b_{m}$

and $a \succcurlyeq^{L} b$ if $a \succ^{L} b$ or $a=b$.

In $\Gamma^{L}$ game a situation $x^{*}=\left(x_{1}^{*}, \ldots, x_{n}^{*}\right) \in X$ is called an equilibrium if for any $i=1, \ldots, n$ fulfills $H_{i}\left(x^{*}\right) \succcurlyeq^{L} H_{i}\left(x^{*} \|_{i} x_{i}\right)$ for every $x_{i} \in X_{i}$.

P. Fishburn [1] defined a lexicographic matrix game and its equilibrium situation in the mixed strategies. He gave an example of such kind of game and showed that an equilibrium situation does not exist in mixed strategies. It is shown by him, that there exists a game where the first player has not got a maximin strategy, and the second - a minimax strategy; there exists a game, where the first player has a maximin strategy, the second has a minimax strategy, but they are different.

V. Podinovski [2] defined a lexicographic finite antagonistic game and explored the problems about the existence of an equilibrium situation in a lexicographic matrix game.Lexicographic noncooperative games with the existence of conditions of an equilibrium situation is studied by $\mathrm{G}$. Beltadze [3,4,5,6,7].

In the process of studying lexicographic cooperative games there exists some problems connected to the existence of a characteristic function and imputation. The existence of $\mathrm{C}$-core in lexicographic cooperative games are studied by G. Beltadze [8]. In the given article we discuss the foundations of lexicographic cooperative games, that is necessary for studying the principles of optimality. We show some differences and similarities in the process of studying them to classical cooperative games. The main part of the paper is studied in the II-V sections. In the second section of the article a lexicographic noncooperative $\Gamma^{L}$ game's characteristic function is discussed and the fairness of its properties is shown. In the third section a lexicographic cooperative game is discussed, as players' lexicographically defined superadditive $m$ dimensional vector function $v=\left(v^{1}, v^{2}, \ldots, v^{m}\right)^{T}$ on $N=\{1,2, \ldots, n\}$ set's subsets. In a lexicographic cooperative game $v=\left(v^{1}, v^{2}, \ldots, v^{m}\right)^{T}$ a concept of imputation is introduced that is given by means of a $m \times n$ dimensional $X$ matrix. The theorem is proved that gives us a full description of $E(v)$ set of $v$ game's imputations. In the fourth section a domination of imputations, cooperative games' isomorphism and lexicographical essential cooperative game are defined. In the fifth section we introduce the definitions of two cooperative games' strategic equivalence, normalization of a lexicographic cooperative game in $(0,1)$ and it is proved that a lexicographic essential cooperative game is strategically equivalent in $(0,1)$ to a normalised cooperative game.

\section{LEXICOGRAPHIC NONCOOPERATIVE GAME'S CHARACTERISTIC FUNCTION}

Let $\quad \Gamma^{L}=<N,\left\{X_{i}\right\}_{i \in N},\left\{H_{i}\right\}_{i \in N}>$ be a lexicographic noncooperative game, for $n$ player's and $m$ dimension. For any $T \subseteq N, T \neq \varnothing$ coalition note that

$H_{T}(x)=\sum_{i \in T} H_{i}(x), x \in X$

If $T=\varnothing$, then suppose, that $H_{T}(x)=\mathrm{O}$ for any $x_{i} \in X_{i}$.

Let define every sets of $T \subseteq N$ coalition mixed strategies by $X_{T}$.

For $T \subseteq N$ coalition let consider a lexicographic antagonistic game $\Gamma_{T}^{L}$, where $T$ and $N \backslash T$ are the players and a function payoff is $H_{T}$.

We consider such $\Gamma^{L}$ games, where exists a lexicographic maximin

$v(T)=\max _{x_{T} \in X_{T}} \min _{x_{N \backslash T} \in X_{N \backslash T}} H_{T}\left(X_{T}, X_{N \backslash T}\right), T \subseteq N$

Definition 2.1. Let $v(T)$ vector-function call a lexicographic noncooperative $\Gamma^{L}$ game's characteristic function.

Some main properties of scalar noncooperative games' characteristic functions are maintained in a lexicographic case. Particularly, such kind of property is superadditivity.

Theorem 2.1. If $T, S \subseteq N$ and $T \cap S=\varnothing$, then $v(T \cup S) \succcurlyeq^{L} v(T)+v(S)$. Such function is called $L$ - superadditival.

Proof. Analogically to the famous result that exists in a classical cooperative game theory we can write the following relations:

$$
\begin{aligned}
& v(T)+v(S)=\max _{x_{T} \in X_{T}} \min _{x_{N \backslash T} \in X_{N \backslash T}} H_{T}\left(X_{T}, X_{N \backslash T}\right)+ \\
& \max _{x_{S} \in X_{S}} \min _{x_{N \backslash S} \in X_{N \backslash S}} H_{S}\left(X_{S}, X_{N \backslash S}\right)=
\end{aligned}
$$




$$
\begin{aligned}
& \max _{X_{T} \times X_{S}}\left(\min _{X_{N \backslash T}} H_{T}\left(X_{T}, X_{N \backslash T}\right)+\min _{X_{N \backslash S}} H_{S}\left(X_{S}, X_{N \backslash S}\right)\right) \\
& { }^{L} \preccurlyeq \max _{X_{T} \times X_{S}}\left(\min _{X_{N \backslash T \backslash S}} H_{T}\left(X_{T}, X_{N \backslash T \backslash S} \times X_{S}\right)\right. \\
& \left.+\min _{X_{N \backslash T \backslash S}} H_{S}\left(X_{S}, X_{T} \times X_{N \backslash S \backslash T}\right)\right)^{L} \\
& \preccurlyeq \max _{X_{T \cup S}}\left(\min _{X_{N \backslash(T \cup S)}} H_{T \cup S}\left(X_{T \cup S}, X_{N \backslash(T \cup S)}\right)=v(T \cup S) .\right.
\end{aligned}
$$

The theorem is proved.

Definition 2.2. We say that a lexicographic noncooperative $\Gamma^{L}$ game's $v$ has a property of complementary, if for all $T \subseteq N$ fulfills the following equality $v(T)+v(N \backslash T)=\mathrm{O}$.

For a scalar zero-sum noncooperative game's characteristic function a property of complementary always takes place. But for a lexicographic zero-sum noncooperative game a property of complementary maybe won't fulfill.

Theorem 2.2. If a lexicographic zero-sum noncooperative game's characteristic function fulfills the property of complementary, then every lexicographic matrix game $\Gamma_{T}^{L}(T \subseteq N)$ has a solution.

Proof. Let $T \subseteq N, \sum_{i \in N} H_{i}=\mathrm{O} \quad$ and $v(T)=-v(N \backslash T)$. Then

$\max _{X_{T}} \min _{X_{N \backslash T}} H_{T}\left(X_{T}, X_{N \backslash T}\right)$

$=-\max _{X_{N \backslash T}} \min _{X_{T}} H_{N \backslash T}\left(X_{N \backslash T}, X_{T}\right)$

$=\min _{X_{N \backslash T}} \max _{X_{T}} H_{T}\left(X_{T}, X_{N \backslash T}\right)$

It means that the lexicographic matrix game $\Gamma_{T}^{L}$ has a solution.

\section{LEXICOGRAPHIC COOPERATIVE GAMES}

Definition 3.1. We call a lexicographic cooperative game in a form of a characteristic function a couple $\langle N, v\rangle$, where $N=\{1,2, \ldots, n\}$ is players' set, $v$ real vector-function on $N$ subset and fulfills the following conditions:

$v(\varnothing)=\mathrm{O} ; \quad v(T \cup S){ }^{L} v(T)+v(S), T, S \subseteq N$, $T \cap S=\varnothing$.

Definition 3.2. If $v(N)=v(S)+v(N \backslash S)$ for any $S \subset N$, then $\langle N, v\rangle$ is called a lexicographic cooperative game with a constant sum.
It is shown by $\mathbf{J}$. von Neumann and O. Morgenstern that every superadditive scalar function $v$, where $v(\varnothing)=0$, is a characteristic function of any zero-sum noncooperative game [9]. Analogical fact for $L-$ superadditive function is proved.

Theorem 3.1. On $N=\{1,2, \ldots, n\}$ set's subsets for every $L$-superadditive function $v_{0}$ we can construct $n$ player's lexicographic noncooperative game $\Gamma^{L}$, where every lexicographic antagonistic $\Gamma_{T}^{L}(T \subseteq N)$ game will have a solution and its meaning will coincide $v_{0}(T)$.

Note, that $N=\{1,2, \ldots, n\} \quad$ set's subset's $L$ - superadditive function $v$ we can be presented by a form of a vector $v=\left(v^{1}, v^{2}, \ldots, v^{m}\right)^{T}$, where $v^{1}$, $v^{2}, \ldots, v^{m}$ functions $N$ subsets are definite scalar functions.

According to this fact that $v$ function is $L-$ superadditive, $v^{1}$ is superadditive too. $v^{2}, \ldots, v^{m}$ functions' maybe won't have analogical property.

Example 3.1. Let discuss a lexicographic cooperative game as a form of a characteristic function $\langle N, v\rangle$, where $N=\{1,2,3\}$, and $v=\left(v^{1}, v^{2}\right)^{T}$ is given as the following form:

$v(\varnothing)=(0 ; 0)^{T}, v(1)=(3 ; 2)^{T}, v(2)=(2 ; 1)^{T}$,

$v(3)=(1 ; 2)^{T}, v(1,2)=(6 ; 2)^{T}$,

$v(1,3)=(4 ; 3)^{T}, v(2 ; 3)=(4 ; 1)^{T}, v(1,2,3)=(8 ; 2)^{T}$.

Here

$v^{2}(\varnothing)=0, v^{2}(1)=2, v^{2}(2)=1, v^{2}(3)=2$, $v^{2}(1,2)=2 \quad, \quad v^{2}(1,3)=3 \quad, \quad v^{2}(2,3)=1 \quad$, $v^{2}(1,2,3)=2$.

It is obvious that $v^{2}$ is not superadditive, because, for example $v^{2}(1,2)<v^{2}(1)+v^{2}(2)$.

It is necessary to discuss a function $\left(v^{2}, \ldots, v^{m}\right)^{T}$ on $N$ subsets, that we note by $v^{*}$. Hence, sometimes we can write $v$ function in the following way $v=\left(v^{1}, v^{*}\right)^{T}$.

Shortly we call $L-$ superadditive function $v=\left(v^{1}, v^{2}, \ldots, v^{m}\right)^{T}=\left(v^{1}, v^{*}\right)^{T}$ a lexicographic cooperative game by $m$ dimension. Because of this, $v^{*}$ function in general is not $L$ - superadditive, that is 
why we can't connect it to a lexicographic cooperative game.

Definition 3.3. In a lexicographic cooperative $v=\left(v^{1}, v^{*}\right)^{T}$ game we call imputation a sequence of $m$ dimensional vectors $X=\left(X_{\cdot_{1}}, X_{\cdot_{2}}, \ldots, X_{\cdot_{n}}\right)$, where fulfills the following conditions:

1. Individual rationality $-X_{\cdot_{i}}{ }^{L} v(i)$ for $i \in N$;

2. Collective rationality $-\sum_{i=1}^{n} X_{\cdot_{i}}=v(N)$.

If we write every $X_{\bullet_{i}}$ vectors in $\left(x_{1 i}, \ldots, x_{m i}\right)^{T}$ column vector form, then in $v=\left(v^{1}, v^{*}\right)^{T}$ game we can write the imputation $X=\left(X_{\cdot_{1}}, X_{\cdot_{2}}, \ldots, X_{\cdot_{n}}\right)$ in a form of matrix

$X=\left(\begin{array}{cccc}x_{11} & x_{12} & \ldots & x_{1 n} \\ x_{21} & x_{22} & \ldots & x_{2 n} \\ \cdot & \cdot & \ldots & \cdot \\ x_{m 1} & x_{m 2} & \ldots & x_{m n}\end{array}\right)$.

Let note the set of imputation in $v=\left(v^{1}, v^{*}\right)^{T}$ game by $E(v)$ and a projection of $E(v)$ set on the $E\left(v^{1}\right)$ set of $v^{1}$ games imputation - by $\operatorname{Pr}\left(E(v) \mid E\left(v^{1}\right)\right)$.

Let observe that if the given notes $X \in E(v)$, then $X^{1}=\left(x_{11}, x_{12}, \ldots, x_{1 n}\right) \in E\left(v^{1}\right)$. Therefore the condition

$E(v) \subseteq E\left(v^{1}\right) \times \mathrm{R}^{n \times(m-1)}$

is correct, where $\mathrm{R}^{n \times(m-1)}$ is a set of matrices

$\left(\begin{array}{ccc}x_{21} & \ldots & x_{2 n} \\ \cdot & \ldots & \cdot \\ x_{m 1} & \ldots & x_{m n}\end{array}\right)$.

Condition (1) means that

$E\left(v^{1}\right) \supseteq \operatorname{Pr}\left(E(v) \mid E\left(v^{1}\right)\right)$.

In fact here the exact equality takes plase.

Theorem 3.2. $E\left(v^{1}\right)=\operatorname{Pr}\left(E(v) \mid E\left(v^{1}\right)\right)$.

Proof. Let hold the argument according to $m$ dimension of $v=\left(v^{1}, v^{*}\right)^{T}$ game's. For $m=1$ an equality is correct, because $v^{1}=v$. Suppose for any $m>1$ and $X^{1}=\left(x_{11}, x_{12}, \ldots, x_{1 n}\right) \in E\left(v^{1}\right)$. Let consider two cases:

1) $x_{1 i}>v^{1}(i)$ for any $i \in N$;

2) $x_{1 i}=v^{1}(i)$ for all $i \in N$.

Let divide 1) case into two subgroups:

1a) $x_{1 i}>v^{1}(i)$ for all $i \in N$;

1b) $\left\{i \in N \mid x_{1 i}=v^{1}(i)\right\} \neq \varnothing$

and

$\left\{i \in N \mid x_{1 i}>v^{1}(i)\right\} \neq \varnothing$.

Suppose we have $1 a)$ case. Let take such $x_{k i}$ $(i=1, \ldots, n ; k=2, \ldots, m)$ numbers and make up a matrix

$$
X^{*}=\left(\begin{array}{ccc}
x_{21} & \ldots & x_{2 n} \\
\cdot & \ldots & \cdot \\
x_{m 1} & \ldots & x_{m n}
\end{array}\right),
$$

for fulfilling the following conditions

$\sum_{i=1}^{n} x_{k i}=v^{k}(N), k=2, \ldots, m$.

Then a matrix

$X=\left(\begin{array}{cccc}x_{11} & x_{12} & \ldots & x_{1 n} \\ x_{21} & x_{22} & \ldots & x_{2 n} \\ \cdot & \cdot & \ldots & \cdot \\ x_{m 1} & x_{m 2} & \ldots & x_{m n}\end{array}\right)=\left(\begin{array}{l}X^{1} \\ X^{*}\end{array}\right)$

will be an imputation in $v=\left(v^{1}, v^{*}\right)^{T}$ game. In fact, a rationality condition $\sum_{i=1}^{n} X_{\cdot_{i}}=v(N)$ comes out from (3) and $\sum_{i=1}^{n} x_{1 i}=v^{1}(N)$ conditions, and individual rationality inequalities $X_{\cdot_{i}} t^{L} v(i)$ comes out from 1a) condition.

In $1 b)$ case let define a matrix $X^{*}$ in the following way. Define

$N_{1}=\left\{i \in N \mid x_{1 i}=v^{1}(i)\right\},\left|N_{1}\right|=n_{1}$,

$N_{2}=\left\{i \in N \mid x_{1 i}>v^{1}(i)\right\}, \quad\left|N_{2}\right|=n_{2}$. 
It is obvious, that here $N_{1} \cup N_{2}=N$, $N_{1} \cap N_{2}=\varnothing$. Let note

$x_{2 i}=v^{2}(i)+1$, if $i \in N_{1}$,

$x_{2 i}=\frac{v^{2}(N)-\sum_{i \in N_{1}} v^{2}(i)-n_{1}}{n_{2}}$, if $i \in N_{2}$.

Let choose other elements in $X^{*}$ matrix from the conditions:

$\sum_{i=1}^{n} x_{k i}=v^{k}(N), k=3, \ldots, m$.

Let us show that the given $X=\left(\begin{array}{c}X^{1} \\ X^{*}\end{array}\right)$ matrix is an imputation in $v=\left(v^{1}, v^{*}\right)^{T}$ game. For $i \in N_{2}$ the condition $X_{._{i}}{ }^{L} v(i)$ comes out from $x_{1 i}>v^{1}(i)$ inequalities. For $i \in N_{1}$ it fulfills that $x_{1 i}=v^{1}(i)$ and $x_{2 i}=v^{2}(i)+1>v^{2}(i)$, from which follows that $X_{\cdot_{i}} \geqslant{ }^{L} v(i)$. So, it fulfills a condition of an individual rationality for $X$.

Let check up that it fulfills an equality $\sum_{i=1}^{n} X_{\cdot_{i}}=v(N)$. If we consider (5) and $\left(x_{11}, x_{12}, \ldots, x_{1 n}\right) \in E\left(v^{1}\right)$ conditions, it is sufficient to check up the equality $\sum_{i=1}^{n} x_{2 i}=v^{2}(N)$. It follows from (4):

$\sum_{i=1}^{n} x_{2 i}=\sum_{i \in N_{1}} x_{2 i}+\sum_{i \in N_{2}} x_{2 i}=\sum_{i \in N_{1}}\left(v^{2}(i)+1\right)+$ $n_{2} \cdot \frac{v^{2}(N)-\sum_{i \in N_{1}} v^{2}(i)-n_{1}}{n_{2}}=$

$\sum_{i \in N_{1}} v^{2}(i)+n_{1}+v^{2}(N)-\sum_{i \in N_{1}} v^{2}(i)-n_{1}=v^{2}(N)$.

Now consider the 2$)$ case. If $x_{1 i}=v^{1}(i)$ for all $i \in N$, then it means that $\sum_{i=1}^{n} v^{1}(i)=v^{1}(N)$. Function $v=\left(v^{1}, v^{*}\right)^{T}$ is $L-$ superadditive and particularly for it should be fulfilled the inequality $v(N) \succcurlyeq{ }^{L} \sum_{i \in N} v(i)$. Hence $v^{1}$ is unessential or $\sum_{i \in S} v^{1}(i)=v^{1}(S)$ for every $S \subseteq N$. According to $v$ 's $L-$ superadditivity we can make a conclusion, that $v^{*}(S) \succcurlyeq^{L} \sum_{i \in S} v^{*}(i)$ for every $S \subseteq N$. Hence $v^{*}$ is $L-$ superadditive function on subsets of $N$ set and its dimension is $m-1$. If we use permissibility of induction in $v^{*}$ game, we can write that $E\left(v^{2}\right)=\operatorname{Pr}\left(E\left(v^{*}\right) \mid E\left(v^{2}\right)\right)$. From here it follows that $E\left(v^{*}\right) \neq \varnothing$. Therefore let take a matrix

$\left(\begin{array}{ccc}x_{21} & \ldots & x_{2 n} \\ \cdot & \ldots & \cdot \\ x_{m 1} & \ldots & x_{m n}\end{array}\right)$

from the sets of $v^{*}$ game's imputation. It is obvious that a matrix

$X=\left(\begin{array}{cccc}v^{1}(1) & v^{1}(2) & \ldots & v^{1}(n) \\ x_{21} & x_{22} & \ldots & x_{2 n} \\ \cdot & \cdot & \ldots & \cdot \\ x_{m 1} & x_{m 2} & \ldots & x_{m n}\end{array}\right)$

gives us the imputation in $v=\left(v^{1}, v^{*}\right)^{T}$ game.

With the help of discussing all cases we have proved that for any $X^{1}=\left(x_{11}, x_{12}, \ldots, x_{1 n}\right) \in E\left(v^{1}\right)$ imputation there exists a matrix $X^{*}$, where $X=\left(\begin{array}{l}X^{1} \\ X^{*}\end{array}\right)$ is an imputation in $v=\left(v^{1}, v^{*}\right)^{T}$ game.

This is equal to the condition

$E\left(v^{1}\right) \subseteq \operatorname{Pr}\left(E(v) \mid E\left(v^{1}\right)\right)$

After taking this and (2) into the consideration we have an exact equality $E\left(v^{1}\right)=\operatorname{Pr}\left(E(v) \mid E\left(v^{1}\right)\right)$ and the theorem is proved. It is shown that in game $v=\left(v^{1}, v^{*}\right)^{T}$ a set of imputation is nonempty.

Now prove a theorem that gives us a full caracterisation of $E(v)$ set. Let note $X^{*}$ matrix's columns by $X_{. i}^{*}(i=1, \ldots, n)$ and for $S \subseteq N$ discuss the following sets

$$
\begin{aligned}
& E_{S}^{0}\left(v^{1}\right)=\left\{X^{1}=\left(x_{11}, \ldots, x_{1 n}\right) \in E\left(v^{1}\right)\right. \\
& \left.\mid x_{1 i}>v^{1}(i), i \notin S ; x_{1 i}=v^{1}(i), i \in S\right\},
\end{aligned}
$$


$E_{S}^{1}\left(v^{*}\right)=\left\{X^{*} \mid \sum_{i=1}^{n} X_{. i}^{*}=v^{*}(N), X_{. i}^{*}{ }^{L} v^{*}(i)\right.$, $i \in S\}$

It is obvious, that if $S=\varnothing$, then

$E_{S}^{1}\left(v^{*}\right)=\left\{X^{*} \mid \sum_{i=1}^{n} X_{. i}^{*}=v^{*}(N)\right\}$.

Theorem 3.3. If $v=\left(v^{1}, v^{*}\right)^{T}$ is a lexicographic cooperative game, then

$E(v)=\bigcup_{S \subseteq N} E_{S}^{0}\left(v^{1}\right) \times E_{S}^{1}\left(v^{*}\right)$.

Proof. It is obvious that

$E\left(v^{1}\right)=\bigcup_{S \subseteq N} E_{S}^{0}\left(v^{1}\right)$.

Therefore, on the basis of the theorem 3.2 it is sufficient to show that for any $X^{1} \in E_{S}^{0}\left(v^{1}\right)$ a set

$$
\left\{X^{*} \mid X=\left(\begin{array}{c}
X^{1} \\
X^{*}
\end{array}\right) \in E(v)\right\}
$$

is equal to $E_{S}^{1}\left(v^{1}\right)$. Let $X^{1} \in E_{S}^{0}\left(v^{1}\right)$, it means that $S=\left\{i \in N \mid x_{1 i}=v^{1}(i)\right\}$. If $X^{*}$ is any kind of matrix, for which $X=\left(\begin{array}{l}X^{1} \\ X^{*}\end{array}\right)$ is an imputation in $v=\left(v^{1}, v^{*}\right)^{T}$ game, then $X_{\cdot_{i}} \succcurlyeq^{L} v(i), i=1, \ldots, n$. As it fulfills an equality $x_{1 i}=v^{1}(i)$ for $i \in S$, therefore $X_{. i}^{*} \succcurlyeq{ }^{L} v^{*}(i)$ for $i \in S$.

On the other hand by the $X^{*}$ definition $\sum_{i \in N} X_{. i}^{*}=v^{*}(N)$. These two relations mean that $X^{*} \in E_{S}^{1}\left(v^{*}\right)$. Hence, when $X^{1} \in E_{S}^{0}\left(v^{1}\right)$ the condition is proved

$\left\{X^{*} \mid X=\left(\begin{array}{c}X^{1} \\ X^{*}\end{array}\right) \in E(v)\right\} \subseteq E_{S}^{1}\left(v^{*}\right)$.

Contrary inclusion is obvious, as from the following conditions $X^{1} \in E_{S}^{0}\left(v^{1}\right)$ and $X^{*} \in E_{S}^{1}\left(v^{*}\right)$ it follows that the matrix $X=\left(\begin{array}{l}X^{1} \\ X^{*}\end{array}\right)$ fulfills the following conditions $\sum_{i \in N} X_{\cdot_{i}}=v(N), X_{\cdot_{i}}{ }^{L} v(i)$, $i=1, \ldots, n$. The theorem is proved.

\section{DOMINATION OF AN IMPUTATION}

For the purpose of studying the principles of optimality in lexicogrsphic cooperative games, as well as in the theory of classical cooperative games, it is necessary to define the apparatus of domination on the set of imputation.

Definition 4.1. We say that from $X, Y \in E(v)$ imputations $X$ dominates $Y$ in $S \subseteq N$ coalition, if

$X_{\cdot_{i}} \succ^{L} Y_{\cdot_{i}}, \quad i \in S$

and

$\sum_{i \in S} X_{\cdot_{i}}{ }^{L} \preccurlyeq v(S)$

We write (6) condition in the following way $X_{S}{ }^{L} Y_{S}$ and (7) condition - $X(S){ }^{L} \preccurlyeq v(S)$. If $X$ dominates $Y$ in $S$ coalition then we write $X \underset{S}{\succ}$.

We say that imputation $X$ dominates an imputation $Y$, if there exists a coalition $S \subseteq N$, for which $X \succ Y$. In this case we write $X \succ Y$.

Analogically to the scalar case, in a lexicographic cooperative $v=\left(v^{1}, v^{*}\right)^{T}$ game domination does not exist in a coalition, that consists of one player or $N$ players.

Truly, from $X \succ Y$ domination it follows that $Y_{\cdot_{i}}{ }^{L} \prec X_{\cdot_{i}}{ }^{L} \preccurlyeq v(i)$, that is against the condition of $X$ imputation's individual rationality. From $X \succ_{N} Y$ follows that $X_{\cdot_{i}} \succ{ }^{L} Y_{{ }_{i}}$ for every $i \in N$ and from here

$\sum_{i \in N} X_{\cdot_{i}} \succ^{L} \sum_{i \in N} Y_{\cdot_{i}}=v(N)$,

that contradicts to the condition of $X$ collective rationality.

Definition 4.2. Let call two lexicographic cooperative $v$ and $u$ games isomorphic, if there exists one-to-one correspondence $\varphi$ between the sets of $E(v)$ and $E(u)$ imputations, that for $X \succ Y$ domination it is sufficient and necessary $\varphi(X) \succ \varphi(Y)$.

Definition 4.3. We call a cooperative $v=\left(v^{1}, \ldots, v^{m}\right)^{T} \quad$ game $\quad L-$ essential, if $v(N) \succ{ }^{L} \sum_{i \in N} v(i)$. If $v^{1}(N)>\sum_{i \in N} v^{1}(i)$ or when a 
game $v^{1}$ is essential, then we call $v$ game completely $L-$ essential.

Theorem 4.1. Every $L$-essential lexicographic $v=\left(v^{1}, \ldots, v^{m}\right)^{T}$ game is isomorphic of a completely $L$ - essential lexicographic game.

Proof. The provement is obvious when $v^{1}(N)>\sum_{i \in N} v^{1}(i)$.

If $\sum_{i \in N} v^{1}(i)=v^{1}(N)$, then by theorem 3.3 in game $v=\left(v^{1}, v^{*}\right)^{T}$ an imputation has the following form $X=\left(\begin{array}{l}X_{0}^{1} \\ X^{*}\end{array}\right)$, where $X_{0}^{1}=\left(v^{1}(1), \ldots, v^{1}(n)\right)$ and $X^{*} \in E\left(v^{*}\right)$.

It is clear that transformation $\varphi: E(v) \rightarrow E\left(v^{*}\right)$ $X=\left(\begin{array}{l}X_{0}^{1} \\ X^{*}\end{array}\right) \rightarrow X^{*}$

is one-to-one. By theorem 3.3 it is a transformation on $E\left(v^{*}\right)$. In $v$ game the condition $X \succ Y$ means that for $i \in S$

$\left(\begin{array}{c}x_{1 i} \\ X_{. i}^{*}\end{array}\right) \succ^{L}\left(\begin{array}{c}y_{1 i} \\ Y_{. i}^{*}\end{array}\right)$ and $\sum_{i \in S}\left(\begin{array}{c}x_{1 i} \\ X_{. i}^{*}\end{array}\right){ }^{L} \preccurlyeq\left(\begin{array}{c}v^{1}(S) \\ v^{*}(S)\end{array}\right)$.

As $y_{1 i}=x_{1 i}=v^{1}(i)$ and $\sum_{i \in S} v^{1}(i)=v^{1}(S)$, we get an equivalent condition

$X_{. i}^{*} \succ^{L} Y_{. i}^{*}, \sum_{i \in S} X_{. i}^{*}{ }^{L} \preccurlyeq v^{*}(S)$,

or in $v^{*}$ game $\varphi(X) \succ_{S} \varphi(Y)$. The theorem is proved.

This theorem shows that in the process of studying every points connected to the lexicographic domination of the imputation is sufficient to be limited by a completely $L$-essential cooperative game.

\section{COOPERATIVE GAME'S NORMALIZATION}

Sometimes it is necessary to compare each other different cooperative game's meanings in one and the same coalition. For this we introduce a concept of a lexicographic cooperative game's normalization. Let define a lexicographic cooperative game's strategic equivalence in advance.

Definition 5.1. For $N=\{1,2, \ldots, n\}$ players' $v=\left(v^{1}, v^{2}, \ldots, v^{m}\right)^{T}$ and $u=\left(u^{1}, u^{2}, \ldots, u^{m}\right)^{T}$ games are said to be strategic equivalent, if there exist such positive numbers $k_{1}, \ldots, k_{m}$ and vectors $\left(c_{i}^{1}, \ldots, c_{i}^{m}\right), i \in N$, that for all $S \subset N$ we have

$\left(v^{1}(S), \ldots, v^{m}(S)\right)^{T}=\left(k_{1} u^{1}(S), \ldots, k_{m} u^{m}(S)\right)^{T}+$ $\sum_{i \in S}\left(c_{i}^{1}, \ldots, c_{i}^{m}\right)^{T}$.

Definition 5.2. A lexicographic cooperative game $v=\left(v^{1}, v^{2}, \ldots, v^{m}\right)^{T}$ is said to be in $(0,1)$ normalized if $\quad v(i)=(0, \ldots, 0)^{T} \quad$ for $\quad$ all $\quad i \in N \quad$ and $v(N)=(1, \ldots, 1)^{T}$.

Theorem 5.1. If in $v=\left(v^{1}, v^{2}, \ldots, v^{m}\right)^{T}$ game $v^{1}, \ldots, v^{m}$ games are essential, or

$v(N)>\sum_{i \in N} v^{j}(i), j=1, \ldots, m$,

then $v=\left(v^{1}, v^{2}, \ldots, v^{m}\right)^{T}$ is strategic equivalent in $(0,1)$ of normalized lexicographic cooperative $u$ game.

Proof. Let find $u=\left(u^{1}, u^{2}, \ldots, u^{m}\right)^{T}$ game with the help of unknown numbers $k_{1}, \ldots, k_{m}$ and vectors $\left(c_{i}^{1}, \ldots, c_{i}^{m}\right), i \in N$ in the following form:

$$
\begin{aligned}
& u(i)=\left(k_{1} v^{1}(i), \ldots, k_{m} v^{m}(i)\right)^{T}+\left(c_{i}^{1}, \ldots, c_{i}^{m}\right)^{T}= \\
& (0, \ldots, 0)^{T}, i \in N, \\
& u(N)=\left(k_{1} v^{1}(N), \ldots, k_{m} v^{m}(N)\right)^{T}+ \\
& \sum_{i \in N}\left(c_{i}^{1}, \ldots, c_{i}^{m}\right)^{T}=(1, \ldots, 1)^{T} .
\end{aligned}
$$

From here we write the systems of equalities:

$$
\begin{aligned}
& \left\{\begin{array}{c}
k_{j} v^{j}(i)+c_{i}^{j}=0 \\
k_{j} v^{j}(N)+\sum_{i \in N} c_{i}^{j}=1,
\end{array}\right. \\
& i \in N ; j=1, \ldots, m .
\end{aligned}
$$

From these systems we get

$$
\begin{aligned}
& k_{j}=\frac{1}{v^{j}(N)-\sum_{i \in N} v^{j}(i)}>0, \\
& c_{i}^{j}=\frac{v^{j}(i)}{\sum_{i \in N} v^{j}(i)-v^{j}(N)},
\end{aligned}
$$

$j=1, \ldots, m ; i \in N$. 
Herewith $k_{j}, j=1, \ldots, m$ and $\left(c_{i}^{1}, \ldots, c_{i}^{m}\right), i \in N$ is defined identically. The theorem is proved

\section{CONCLUSION}

A lexicographic cooperative game is defined as a cooperative game with vector-functions of a finite dimension's payoffs. A vector's coordinates are ranked by decreasing. In such cooperative games some principles of optimality of a scalar cooperative games maybe will not be fulfilled. Basic foundations of lexicographic cooperative games are offered.

\section{REFERENCES}

[1] P. C. Fishburn, "On the foundations of game theory: the case of non-Arximedean utilites", Internat. J. Game Theory, 1, № 2 (1972), pp. 65-71.

[2] V. V. Podinovski, "Lexicographic games", Advances in game theory (Proc. Second All- Union Conf. Vilnius 1971), Izdat. "Mintis", Vilnius, (1973). pp. 100-103 (in Russian).

[3] G.N. Beltadze, "A mixed extension of finite noncoalition lexicographic games", Bulletin of the Academy of sciences of the Georgian SSR, 98, № 2 (1980), pp. 273-276 (in Russian).

[4] G.N. Beltadze, J.A.Giorgobiani, "Metastrategic extensions of Lexicographic Noncooperative Gamein case of two players", Bulleten of the Georgian National Academy of Sciences, vol 2, no 2, 2008, pp. 9-13.

[5] M. Salukvadze, G. Beltadze and F. Criado, "Dyadic theoretical games models of decision making for the lexicographic vector payoffs", International Journal of Information Technology and Decision Making, Vol. 8, Issue 2, 2009, pp. 193-216.

[6] G. N Beltadze, "Lexicographic noncooperative game's mixed extension with criteria", International Journal of Systems and Software, Asian Research Publishing Network (ARPN) Publishers, Vol 1, № 8, November 2011, pp. 247 250.

[7] G.N. Beltadze, "Lexicographic Multistage Games With Perfect Information", Informational and Communication Technologies - Theory and Practice: Proceedings of the International Scientific Conference, ICTMC-2010 Devoted to the 80th Anniversary of I.V. Prangishvili, Nova Publishers, 664 p. USA, 2012, pp. 275-281.

[8] G.N. Beltadze, "Cooperative games with lexicographic payoffs", Bulletin of the Academy of sciences of the Georgian SSR, 99, № 2 (1980), pp. 309 -312 (in Russian).
[9] J. von Neuman, O. Morgenstern, "Theory of Games and Economic Behavior", Prinston University Press, 1944.

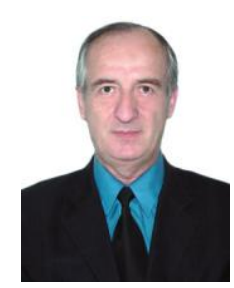

MR. Guram N. Beltadze is a full Professor, mathematician at Informatics and Control Systems Faculty. He finished Tbilisi State University. He got a postgraduate education in the Academy of Sciences of the USSR in St.-Petersburg. His superrvisor was prof. N.N. Vorobiev. 1982 - Ph.D. at St.-Petersburg State University, 1992 - Dr. of Sci. of St.Petersburg State University. Fields of research: Game Theory, Operations Research. 